\title{
A Statistical Model for Interpreting Computerized Dynamic Posturography Data
}

\author{
Alan H. Feiveson*, E. Jeffrey Metter, and William H. Paloski, Member, IEEE
}

\begin{abstract}
Computerized dynamic posturography (CDP) is widely used for assessment of altered balance control. CDP trials are quantified using the equilibrium score (ES), which ranges from zero to 100, as a decreasing function of peak sway angle. The problem of how best to model and analyze ESs from a controlled study is considered. The ES often exhibits a skewed distribution in repeated trials, which can lead to incorrect inference when applying standard regression or analysis of variance models. Furthermore, CDP trials are terminated when a patient loses balance. In these situations, the ES is not observable, but is assigned the lowest possible score-zero. As a result, the response variable has a mixed discrete-continuous distribution, further compromising inference obtained by standard statistical methods. Here, we develop alternative methodology for analyzing ESs under a stochastic model extending the ES to a continuous latent random variable that always exists, but is unobserved in the event of a fall. Loss of balance occurs conditionally, with probability depending on the realized latent ES. After fitting the model by a form of quasi-maximum-likelihood, one may perform statistical inference to assess the effects of explanatory variables. An example is provided, using data from the NIH/NIA Baltimore Longitudinal Study on Aging.
\end{abstract}

Index Terms-Balance control, beta distribution, equilibrium score, latent response, maximum-likelihood, mixed distribution, nonresponse, posture test.

\section{INTRODUCTION}

\section{A. Background}

C OMPUTERIZED dynamic posturography (CDP) has been shown to provide a clinically useful, quantitative assessment of altered balance control function in patients having central or peripheral vestibular dysfunction of various etiologies [1], [2]. The standard clinical test battery provided by the Equitest CDP system (Neurocom International, Clackamas, OR) comprises a set of motor control tests that quantify the patient's biomechanical responses to sudden base-of-support perturbations and a set of sensory organization tests (SOTs) that quantify the patient's ability to maintain upright balance

Manuscript received June 1, 2001; revised November 28, 2001. This work was supported in part by the National Aeronautics and Space Administration (NASA), in part by the National Institute of Health (NIH) under Grant 00205, and in part by the NIH/National Institute of Aging (NIA)/Baltimore Longitudinal Study on Aging under Project 1Z01AG00014-41. Asterisk indicates corresponding author.

*A. H. Feiveson is with the National Aeronautics and Space Administration, Johnson Space Center, mail code SD3, Houston, TX 77058 USA. (e-mail: alan.h.feiveson1@jsc.nasa.gov).

E. J. Metter is with the National Institute of Aging/Laboratory of Clinical Investigation, Gerontology Research Center, Baltimore, MD 21224-2780 USA.

W. H. Paloski is with the National Aeronautics and Space Administration, Johnson Space Center, Houston, TX 77058 USA.

Publisher Item Identifier S 0018-9294(02)02900-7.

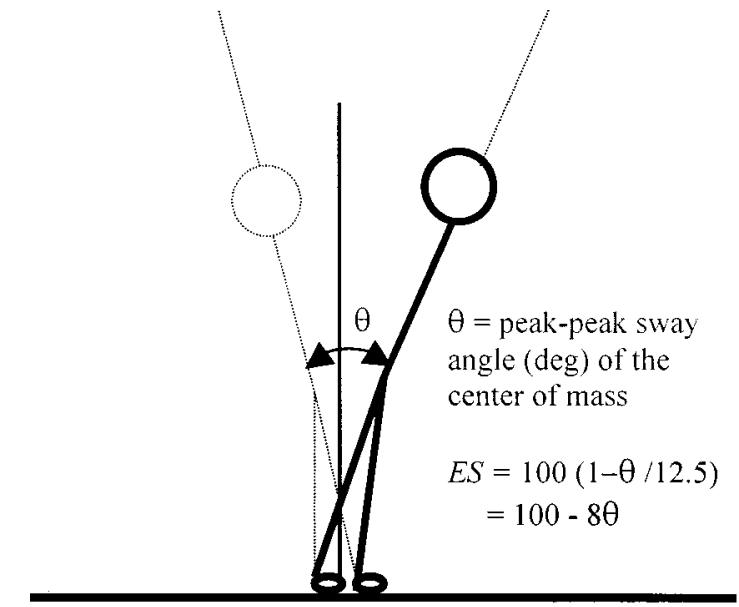

Fig. 1. Schematic of CDP platform and definition of equilibrium score (ES).

with normal, absent, and/or mechanically altered visual and proprioceptive sensory inputs.

The SOT battery consists of a subject standing on a support platform while undergoing three 20 -s trials of each of the following test conditions: SOT 1) standard eyes open Romberg test-visual surround and platform fixed; SOT 2) standard eyes closed Romberg test-platform fixed; SOT 3) sway-referenced visual surround with fixed platform; SOT 4) sway-referenced platform with fixed visual surround; SOT 5) sway-referenced platform with eyes closed; and SOT 6) sway-referenced platform with sway-referenced visual surround. The primary dependent measure is the "equilibrium score" (ES), a performance measure ranging from zero to 100 , which is normally a decreasing linear function of a peak center-of-gravity sway angle over each 20-s trial (Fig. 1). However during some CDP trials a patient loses balance, in which case the peak sway angle is not observable. In such trials, the ES is conventionally set equal to the minimum score of zero (Section II-B). In the remainder of this paper, we will refer to this situation as a "fall," although in practice, subjects either take a step or are physically restrained to prevent actual falling.

\section{B. Interpreting Change}

Assuming that fundamental levels of balance control performance are manifested in the ES, how should ES data be used to test the effects of possible explanatory variables? In our models, we assume that every subject approaches the test platform with a current level of skill for integrating sensory information that in turn drives balance control performance. However, even under rigidly controlled conditions, this a priori skill level varies randomly from subject to subject. Furthermore, actual performance on the CDP battery, reflected in the ES, varies over multiple 


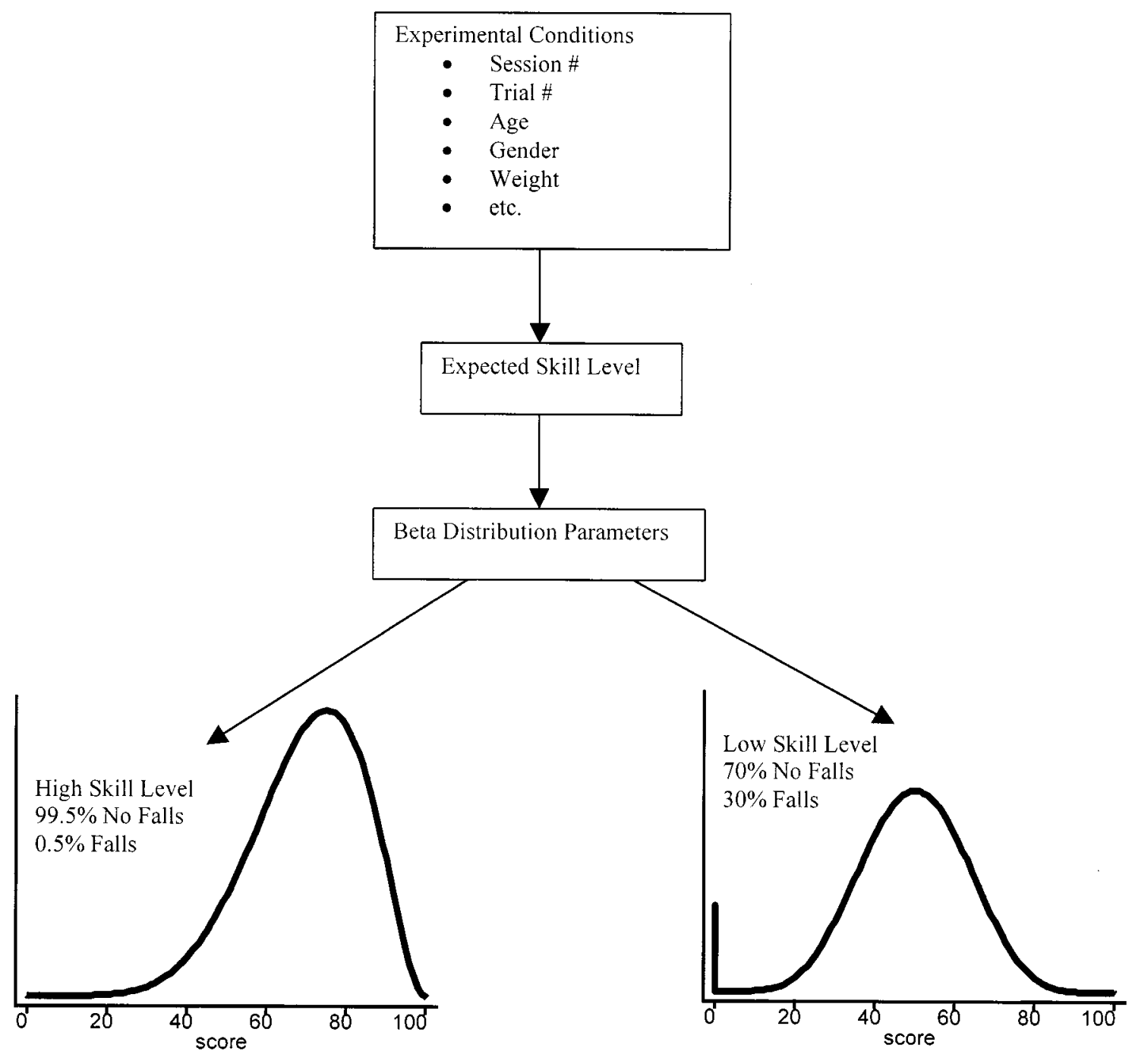

Fig. 2. Expected skill level and distribution of equilibrium scores.

trials of the test performed by the same subject. As a result, we model the ES as a random variable whose probability distribution is related to the current expected skill level. In a designed experiment, the skill level would in turn be affected by values of one or more experimental variables (Fig. 2).

With an experimental design that includes repeated CDP test sessions on individual subjects over a period of time, possibly with replicated trials per session, changes attributed to explanatory variables should be evaluated relative to withinand between-subject variation using appropriate methods of statistical inference. Typically, past studies have reported changes in mean ES or peak-peak sway angles under various conditions [3], [4]; however the sway angle or the ES often exhibits a skewed distribution (see examples in Section II) causing estimates of the mean to be unduly influenced by outliers. Under these circumstances, use of regression or analysis of variance models, which assume homoscedastic normally distributed observations about a deterministically modeled mean, may produce misleading conclusions about the effects of explanatory variables on the ability to control balance. Some obviation of nonnormality through data transformation may be possible, but it is unlikely that all multilevel random error sources (subjects, sessions, trials) in a typical repeated measures design could be modified acceptably by a single transformation. In any event, no data transformation could correct for the failure to obtain a continuous distribution for the ES when a subject falls.

In Section IV, we present an example analysis using a more promising approach to analyzing ESs with a family of beta distributions when there is no fall, and then modifying these distributions with an adjustment that accounts for the probability of a fall. Motivation for the beta distributional model is provided in Section II. In Section III, we describe the method of parameter estimation used to fit the model. Finally in Section V, we discuss the rationale for choosing our method as well as limitations and possible alternatives.

\section{Distributional Models For EQuilibrium Scores}

\section{A. Population-Averaged Models}

We concentrate here on the problem of assessing the average effects of experimental variables over a large population of potential subjects, as opposed to characterizing the statistical mechanism of how subjects and repeated observations vary. 


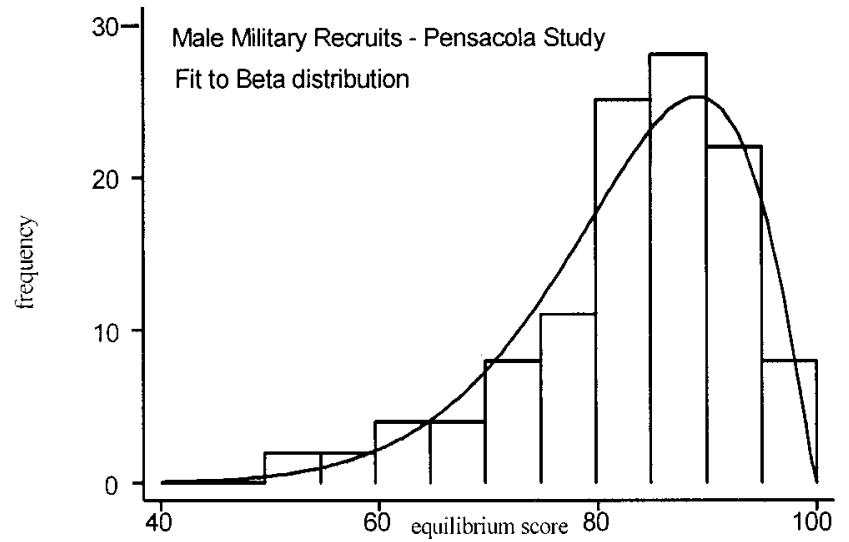

(a)

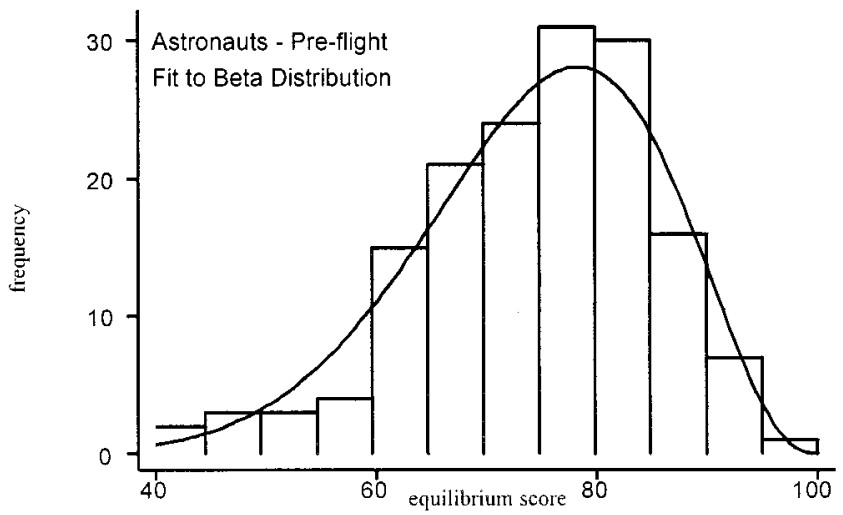

(b)

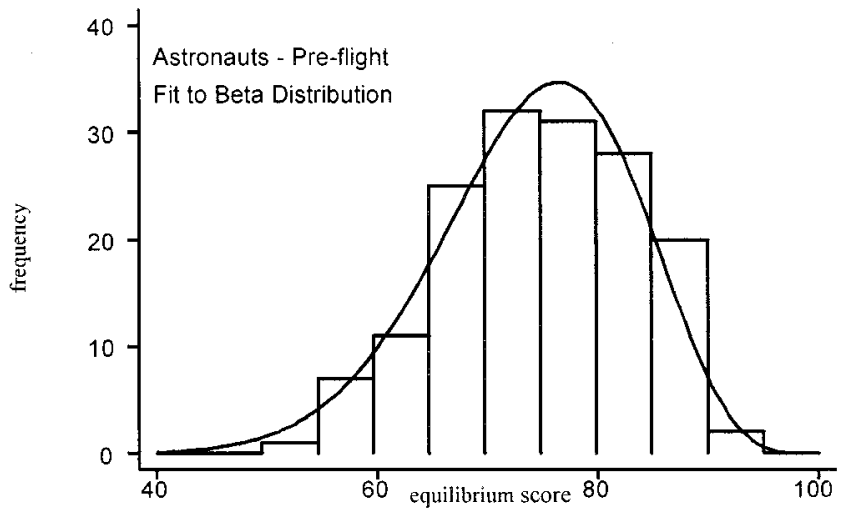

(c)

Fig. 3. (a) Histogram of equilibrium scores for 114 trials of SOT 6 performed by 19 male marine recruits, with best-fitting beta distribution. (b) Histogram of equilibrium scores for 159 trials of SOT 6 performed by 34 astronauts prior to flight, with best-fitting beta distribution. (c) Histogram of equilibrium scores for 159 trials of SOT 5, performed by 34 astronauts prior to flight, with best-fitting beta distribution.

Therefore, we propose using parametric population-averaged models for interpreting changes in the ES. For a given set of covariate values, these marginal models estimate the distribution of scores one would obtain over a population of individuals, with each performing the test exactly once. For a good discussion of population-averaged vs. subject-specific models, see [5]. After fitting a population-averaged model, robust estimators of variance [6], [7] can be used to adjust standard errors of parameter estimates to account for dependence due to repeated observations on individuals.

\section{B. Model Form-No Falls}

The equilibrium score, say $Y$, is defined by $Y=100-8 \theta$, where $\theta$ is a continuous peak-peak sway angle (in degrees) observed only for tests on which a subject does not fall (Fig. 1). In practice, $\theta$ rarely exceeds $10^{\circ}$ and is theoretically limited to a value of about $12.5^{\circ}$ [8], [9], the latter being the approximate angle after which the subject's center of gravity would be outside the region of support. As a result, $Y$ is bounded between zero and 100. When conditions are benign (as when falls are unlikely), $Y$ often has a skewed distribution because of a preponderance of scores near the maximum of 100 . We, therefore, propose to model its marginal probability distribution, when there is a negligible probability of a fall, with a scaled beta distribution, whose parameters are specified functions of the covariates.

This choice of model was reinforced by observation of empirical distributions of ES obtained from two studies that tested fairly homogeneous groups of subjects under similar conditions (hence, the assumption of a constant covariate pattern). Three illustrative examples from these studies comparing histograms with best-matching beta densities follow. The first example is for SOT 6 performed by 19 male Marine and Navy recruits [10]. Although each subject was tested three times each on five sequential days ("sessions"), only data from the last two sessions were used to ensure that learning effects did not significantly alter the distribution of scores. For each session, a subject performed the test three times, making a total of 114 observations. Data for the second and third examples consists of SOT 6 and SOT 5 ES obtained from a study of 34 NASA astronauts prior to flight [11]. Tests were performed on multiple sessions of three trials each per subject with data for early test sessions being discarded, leaving a total of 157 (SOT 5) and 159 (SOT 6) observations. There were no falls in any of these examples.

For a fixed covariate pattern with repeated measurements on a random sample of individuals, an unbiased estimate of the marginal probability $P(S)$, of an ES being within a prespecified measurable subset $S$ of $(0, \infty)$, can be obtained by simple counting. Let $y_{i j}$ be the observed score for the $j$ th measurement on the $i$ th subject and let the indicator variable $a_{i j}=1$ if $y_{i j} \in S$; otherwise, $a_{i j}=0$. If all $y_{i j}$ have a common marginal distribution, then $P(S)$ is estimated unbiasedly by the average of $a_{i j}$ over all $i$ and $j$ regardless of dependence between observations pertaining to the same subject. It follows that any histogram constructed from the $y_{i j}$ would be an unbiased estimate of the true corresponding marginal bin probabilities as long as the bins were specified in advance.

Fig. 3(a) shows a histogram of the 114 observations of the SOT 6 ES measured on the 19 military trainees, with a superimposed best-fitting scaled beta distribution. Although the observations are not independent, coming from only 19 subjects, the distribution of ESs is clearly skewed, as is the matching beta density. In Fig. 3(b), a similar plot of a histogram and superimposed scaled beta density is shown for SOT 6 ESs from the astronaut data. Again, there is an indication of skewness. Clearly, normal distributions would not be good models for these examples. However for other cases, a normal distribution might be a good approximate model. Even so, the beta distribution, which can approach normality for certain ranges of its parameters, is flexible enough to also be used as an effective universal model. An example of near-normality is shown in Fig. 3(c) for SOT 5 
from the astronaut data. In Section II-C, we develop a rationale for treating falls as events whose probability of occurrence is linked to the same beta distribution that drives ESs when falls do not occur.

\section{Falls}

When a fall occurs, the peak-peak sway angle becomes meaningless; we know only that the subject's sway angle exceeded controllable limits. Past treatments of falls in statistical analyses have been to either discard them [12], or to use analysis methodology intended for continuous data after setting $\theta$ for falls equal to the theoretical maximum of $12.5^{\circ}$ [3], [4], [13], [14]. We search for improvements to both of these approaches, because 1) discarding falls would effectively ignore information on the most extreme cases of instability, the very type of response we would often attempt to predict as a function of independent design or explanatory variables or 2) as already noted, assigning a fixed value for falls and analyzing as though $\theta$ were a continuous response variable would render conventional statistical methods of inference such as analysis of variance, even more inappropriate than they would be under a nonnormal continuous distribution. These problems would hold equally for the ES.

Here, we also adopt the convention of setting $\theta=12.5$ (i.e., $Y=0$ ) when there is a fall, but we make the modification of analyzing $Y$ as a mixed continuous-discrete random variable (which it is), rather than as one continuous random variable. First, we assume that prior to a SOT test, an average subject's expected skill level can be quantified by a "stability" index $\mu$, reflecting experimental conditions or the type of SOT administered. If falls were not possible, $\mu$ would be the mean ES over a population of subjects; however by thinking of $\mu$ as an index of stability rather than as a transformed sway angle, we can extend its definition to the case of falls. For each test resulting in a fall, it is reasonable to assume that conditions were such that the expected skill level was low, but no quantitative estimate of the numerical index $\mu$ was available. Nevertheless, we can conceptualize $\mu$ to be a decreasing function of the expected skill level until in the limiting situation, the case $\mu=0$ would correspond to every subject being unable to stand.

Second, we tie the event of a fall to the skill level, but only in a probabilistic sense. Let $\phi$ be a binary indicator of a fall; i.e., $\phi=1$ if a fall occurs, otherwise $\phi=0$. To complete the model, we first write the equilibrium score $Y$ as

$$
Y= \begin{cases}Y^{*}, & \phi=0 \\ 0, & \phi=1\end{cases}
$$

where $Y^{*}$ is a continuous random variable, which is the same as the ES when there is no fall, and whose population-averaged mean is $\mu$. We shall refer to $Y^{*}$ as the latent ES in the sense that it is not always observed, but when it is, it is identical to $Y$. We then model $P\left(\phi=1 \mid Y^{*}=y\right)$ by the function $G(y)=(1-y / 100)^{r}$ which approaches 1 as $y \rightarrow 0$, where $r$ is an unknown parameter to be estimated, possibly being a function of concomitant variables such as a subject's age. For example, Fig. 4 shows a graph of $G(y)$ for $r=3.0$. The implication of this model is that even for a fixed a priori skill level, falls do not necessarily occur with every trial of the test; however falls become more likely as performance (quantified by $Y^{*}$ ) degrades. Since $Y^{*}=Y$ when there is no fall, and because a

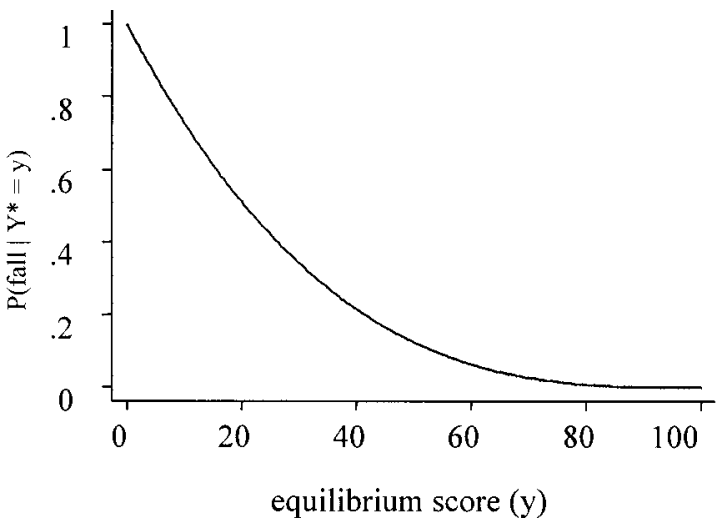

Fig. 4. Illustration of conditional probability of a fall as a function of the latent equilibrium score $y(r=3.0)$.

scaled beta distribution appears to fit the marginal distribution of $Y$ when the probability of falls is negligible (as in the Marine or astronaut examples), we propose to use a scaled beta distribution for $Y^{*}$ in the general situation.

In Section III, we show how this model may be fit to data consisting of observations of $Y$, defined by (1), when falls are present. In so doing, use is made of all the data. Although $Y^{*}$ is not observed with falls, such cases contribute information about the beta parameters through the function $G$. Once the estimated distribution of $Y^{*}$ is obtained, any of its characteristics (mean, median, extreme percentage points, etc) can be expressed in terms of experimental variables. With the additional estimation of the fall function $G$, the probability of a fall can also be expressed in terms of experimental variables. Conversely, statistical inference can be made about the effect of the experimental variables on the distribution of $Y^{*}, Y$, and the fall function.

\section{ESTIMATION OF PARAMETERS}

We now describe an approximate maximum-likelihood parameter estimation process as applicable to a situation in which the actual ES, defined by (1), has been obtained at various points in time for $N$ subjects. The method is approximate in that the likelihood function maximized is the one that would obtain if all observations were independent. Let $y_{i j}$ and $\phi_{i j}$ be the respective ES and fall indicator for the $j$ th test on the $i$ th subject $\left(j=1, \ldots, N_{i}\right)$ and let $\boldsymbol{x}_{i j}$ be a vector of concomitant information thought to affect the distribution of $y_{i j}$. Recall that in Section II-C, we assumed the marginal distribution of the corresponding latent ES, $y_{i j}^{*}$, is that of a scaled beta distribution. Specifically, if $f\left(y_{i j}\right)$ is the marginal density evaluated at $y_{i j}$, then

$$
f\left(y_{i j}\right)=\frac{1}{100} \frac{\Gamma(p+q)}{\Gamma(p) \Gamma(q)}\left(\frac{y_{i j}}{100}\right)^{p-1}\left(1-\frac{y_{i j}}{100}\right)^{q-1}
$$

where in general, the parameters $p>0$ and $q>0$ depend on $\boldsymbol{x}_{i j}$. Here, we have chosen to represent $\log p$ and $\log q$ as linear functions of $\boldsymbol{x}_{i j}$; i.e., $\log p=\alpha^{\prime} \boldsymbol{x}_{i j}$ and $\log q=\beta^{\prime} \boldsymbol{x}_{i j}$ where $\alpha$ and $\beta$ are unknown vectors of coefficients to be estimated. The mean of the scaled beta distribution is $100 p /(p+q)$, which in general depends on $\boldsymbol{x}_{i j}$. For $p>q$, the mean exceeds 50 and approaches the perfect score of 100 as $p$ increases for fixed $q$. Illustrations of some scaled beta densities are shown in Fig. 5 for the example of Section IV. 


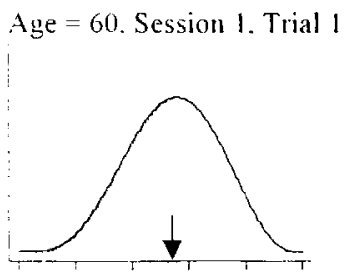

Age $=60$. Session 2 Trial 1

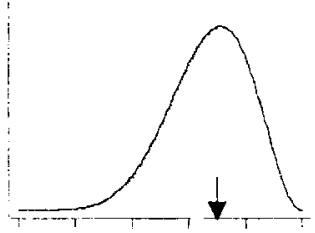

$\begin{array}{lllll}0 & 20 & 40 & 60 & 80100\end{array}$
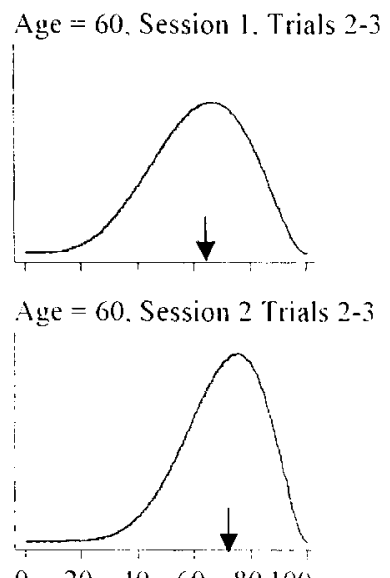

$\begin{array}{llllll}0 & 20 & 40 & 60 & 80100\end{array}$
Age $=60$. Session 2 Trials $2-3$

equilibrium score

Fig. 5. Example of scaled Beta distributions. Estimated densities of latent equilibrium scores, by session and trial number for a 60 -yr.-old subject in the BLSA study (see Section IV). Arrows indicate means of distributions.

In our fall model (Section II), we assume that conditionally on $Y_{i j}^{*}=y$, falls occur with probability $G(y)=(1-y / 100)^{r}$. The unconditional probability of a fall, say $\pi_{i j}$, is then equal to $\int_{0}^{100} G(y) f(y) d y$. Also allowing $\log r$ to be a linear function of $\boldsymbol{x}_{i j}$, say $\log r=\gamma^{\prime} \boldsymbol{x}_{i j}$, it can be shown that in terms of $\alpha, \beta$, and $\gamma$

$\pi_{i j}=$

$$
\frac{\Gamma\left(\exp \left(\gamma^{\prime} x_{i j}\right)+\exp \left(\beta^{\prime} x_{i j}\right)\right) \Gamma\left(\exp \left(\alpha^{\prime} x_{i j}\right)+\exp \left(\beta^{\prime} x_{i j}\right)\right)}{\Gamma\left(\exp \left(\beta^{\prime} x_{i j}\right)\right) \Gamma\left(\exp \left(\gamma^{\prime} x_{i j}\right)+\exp \left(\alpha^{\prime} x_{i j}\right)+\exp \left(\beta^{\prime} x_{i j}\right)\right)} .
$$

The conditional density of $Y_{i j}^{*}$ (and also of $Y_{i j}$ ) evaluated at the point $y_{i j}$ when there is no fall, is $\left(1-G\left(y_{i j}\right)\right) f\left(y_{i j}\right) /(1-$ $\left.\pi_{i j}\right)$; hence, the $\log$ likelihood function assuming independent observations would be of the form

$$
\log L=C+\sum_{i, j} Z_{i j}
$$

where $C$ is a constant, and where

$$
\begin{array}{r}
Z_{i j}=\left(1-\phi_{i j}\right)\left[\log \left(1-G\left(y_{i j}\right)\right)+\log \left(f\left(y_{i j}\right)\right)\right] \\
+\phi_{i j} \log \pi_{i j} .
\end{array}
$$

Our approach was to estimate the coefficient vectors $\alpha, \beta$, and $\gamma$ through maximization of $\log L$; i.e., by solving the system of equations

$$
\frac{\partial}{\partial \alpha} \log L=\frac{\partial}{\partial \beta} \log L=\frac{\partial}{\partial \gamma} \log L=0 .
$$

For experimental designs with multiple observations on each subject, one could not assume independent observations. However the vectors $\hat{\alpha}, \hat{\beta}$, and $\hat{\gamma}$ which satisfy (6) are examples of "M"-estimators (for example, see [15]), which in our setting can be shown to be asymptotically normal and consistent estimators of $\alpha, \beta$ and $\gamma$ as the number of subjects $(N)$ increases [16]. Statistical inference is based on the approximate normality of these quasi-maximum-likelihood estimates, using the robust estimator of their covariance matrix [7]. The latter is calculated from within-cluster sums of the score vectors
TABLE I

SUMMARY OF BLSA FALLS, BY AGE BRACKET

\begin{tabular}{l|rrr}
\hline & \multicolumn{3}{|c}{$n o$} \\
Age & fall fall & total \\
\hline $20-29$ & 66 & 3 & 69 \\
$30-39$ & 41 & 1 & 42 \\
$40-49$ & 125 & 4 & 129 \\
$50-59$ & 118 & 14 & 132 \\
$60-69$ & 65 & 13 & 78 \\
$70-79$ & 85 & 20 & 105 \\
$>79$ & 21 & 3 & 24 \\
All & 521 & 58 & 579 \\
\hline
\end{tabular}

$\left(\partial / \partial \alpha \log L_{i j}, \partial / \partial \beta \log L_{i j}, \partial / \partial \gamma \log L_{i j}\right)^{\prime}$ to account for the dependent observations pertaining to each subject. For the example of Section IV, the estimation procedure was programmed and run in Stata [17]. We found this software especially well-suited for our problem, because its maximum-likelihood optimization package has a built-in capability for robust variance estimation; however any programmable optimization package could have been used. A good introductory discussion of the robust estimator may be found in the Stata maximum-likelihood reference manual [18].

\section{RESULTS}

To illustrate the proposed analysis methodology, we give an example in which we estimate the amount of age-adjusted improvement in SOT 6 scores due to learning over repeated performance of the test by a self-selected general population as part of the NIH/NIA Baltimore Longitudinal Study of Aging (BLSA) [19]. In this analysis, 102 BLSA subjects ranging in age from 22 to 88 years (Table I) performed SOT 6 on consecutive days ("Session 1" and "Session 2"), with three repeated trials of the test on each day. Of the 579 SOT 6 test trials, 58 resulted in falls. Using the model of Section III, we tested for effects of session number, trial number and/or age on the distribution of the ES. It was hypothesized that subjects would perform better on their second session, i.e., after having experienced a full day of testing, and that within a session, there would be an incremental learning effect over the three trials. In addition, we expected an overall decrement in performance for older subjects, with the ability to acclimate to the test also possibly being affected by age.

After some experimentation representing $\log p, \log q$, and $\log r$ as linear functions of session number trial number, subject age, and possible interactions, the most parsimonious model which adequately explained the data was found to be

$$
\begin{aligned}
& \log p=\alpha_{0}+\alpha_{1} \delta_{S 1} \\
& \log q=\beta_{0}+\beta_{1} \delta_{T 1}+\beta_{2} \delta_{T 1} \delta_{S 1} \\
& \log r=\gamma_{0}+\gamma_{1} A G E+\gamma_{2} \delta_{S 1} A G E
\end{aligned}
$$

where $\delta_{S 1}$ and $\delta_{T 1}$ are dummy variables indicating Session 1 and Trial 1, respectively, and where $A G E$ is the age of the subject (in years). Output from the estimation performed by STATA is shown in Table II.

Table II gives estimated values of a) $\alpha_{0}$ and $\alpha_{1}$ in the " $\log p$ " block, b) $\beta_{0}, \beta_{1}$, and $\beta_{2}$ in the " $\log q$ " block, and c) $\gamma_{0}, \gamma_{1}$, and $\gamma_{2}$ in the " $\log r$ " block. All standard errors are calculated 
TABLE II

STATA MAXIMUM-LIKELIHOOD ANALYSIS RESULTS NO. OF OBSERVATIONS $=579$ LOG LIKELIHOOD $=129.34$

\begin{tabular}{|c|c|c|c|c|c|c|c|}
\hline \multirow[t]{2}{*}{$\log p$} & & Coef. & $\begin{array}{l}\text { Robust } \\
\text { Std. Err. }\end{array}$ & Z & $\mathbf{P}>\mathbf{z}$ & $\begin{array}{c}95 \% \\
\text { Confidence }\end{array}$ & Interval \\
\hline & $\alpha_{1}$ & -0.367 & 0.074 & -4.96 & $<0.001$ & -0.512 & -0.223 \\
\hline & $\alpha_{n}$ & 1.952 & 0.087 & 22.56 & $<0.001$ & 1.782 & 2.121 \\
\hline \multicolumn{8}{|l|}{$\log 9$} \\
\hline & $\beta_{1}$ & 0.144 & 0.061 & 2.36 & 0.018 & 0.025 & 0.263 \\
\hline & $\beta_{2}$ & 0.167 & 0.073 & 2.28 & 0.022 & 0.024 & 0.31 \\
\hline & $\beta_{t}$ & 1.091 & 0.084 & 12.97 & $<0.001$ & 0.926 & 1.256 \\
\hline \multicolumn{8}{|l|}{$\log r$} \\
\hline & $\because$ & -0.0163 & 0.0065 & -2.5 & 0.012 & -0.0291 & -0.0035 \\
\hline & $y_{2}$ & -0.0093 & 0.0027 & -3.49 & $<0.001$ & -0.0146 & -0.0041 \\
\hline & $y_{1}$ & 2.2422 & 0.3396 & 6.6 & $<0,001$ & 1.5768 & 2.9084 \\
\hline
\end{tabular}

TABLE III

ESTIMATES OF BETA PARAMETERS $p$ AND $q$ By SESSION AND TRIAL NUMBER

\begin{tabular}{l|ll}
\hline & Trial 1 & Trials 2-3 \\
\cline { 1 - 1 } Session 1 & & \\
\cline { 1 - 1 }$p$ & 4.88 & 4.88 \\
$q$ & 4.07 & 2.98 \\
\hline Session 2 & & \\
\cline { 1 - 1 }$p$ & 7.04 & 7.04 \\
$q$ & 3.44 & 2.98 \\
\hline
\end{tabular}

allowing for within-subject correlation. A comparison with corresponding standard errors shows all of the parameter estimates were significantly different from zero $(P<0.025)$; however subsequent inclusion of terms for age in the equations for $\log p$ and $\log q$ did not produce a significant improvement to the model fit. With the parameterization of the beta distribution used here, larger values of $p$ and smaller values of $q$ lead to increased latent ESs (better balance control). Therefore, the significant negative value of $\alpha_{1}(P<0.001)$ indicates a definite tendency for overall lower performance on the first day of testing. In addition, performance was somewhat worse on the first of the three trials $\left(\beta_{1}>0 ; P=0.018\right)$ regardless of session number, with the effect being somewhat exacerbated during Session $1\left(\beta_{2}>0 ; P=0.022\right)$. Since there was no evidence of any effect of Trial 3 as compared with Trial 2, parameter estimates for both of these trials were constrained to be equal. Table III shows the estimates of $p$ and $q$ for each session and trial. To illustrate their effect, estimates of the mean $\mu$ and respective scaled beta densities for the latent ES are shown in Fig. 5 for the distinct session-trial combinations and for a typical subject of age $60 \mathrm{yrs}$. Note how the distributions shift toward improved performance for Session 2 relative to Session 1 and for Trials 2 and 3 relative to Trial 1.

In order to relate the distribution of latent ESs to that of actual ESs with falls, the probability of a fall needs to be considered in accordance with the model of Section II-C. Because $G(y)=P\left(\right.$ fall $\left.\mid Y^{*}=y\right)=(1-y / 100)^{r}$, lower values of $r$ correspond to an increased conditional probability of falling for a given amount of sway. In the equation for $\log r, \gamma_{1}$, the coefficient of age was a significantly less than zero, indicating smaller values of $r$ and, hence, an increased conditional fall probability for older people $(P=0.012)$. This effect was even stronger for

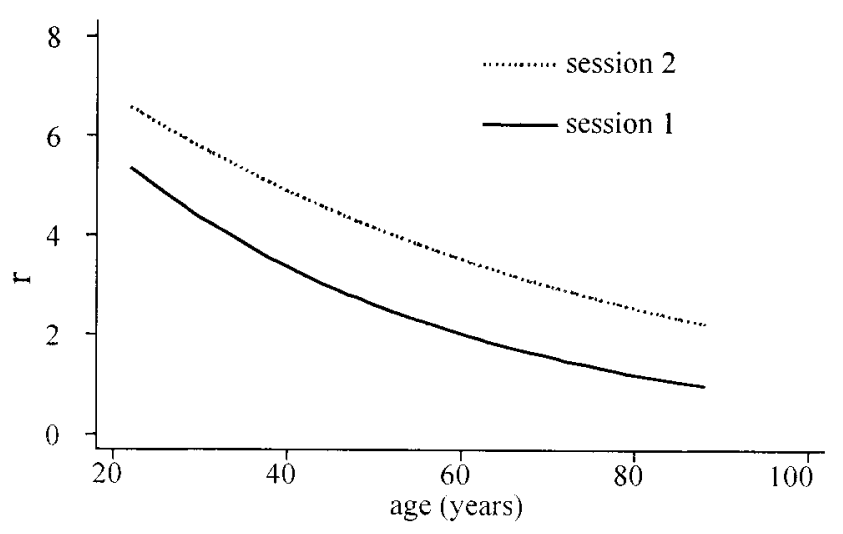

Fig. 6. Estimated fall model parameter $r$ as a function of age-Sessions 1 and 2 .

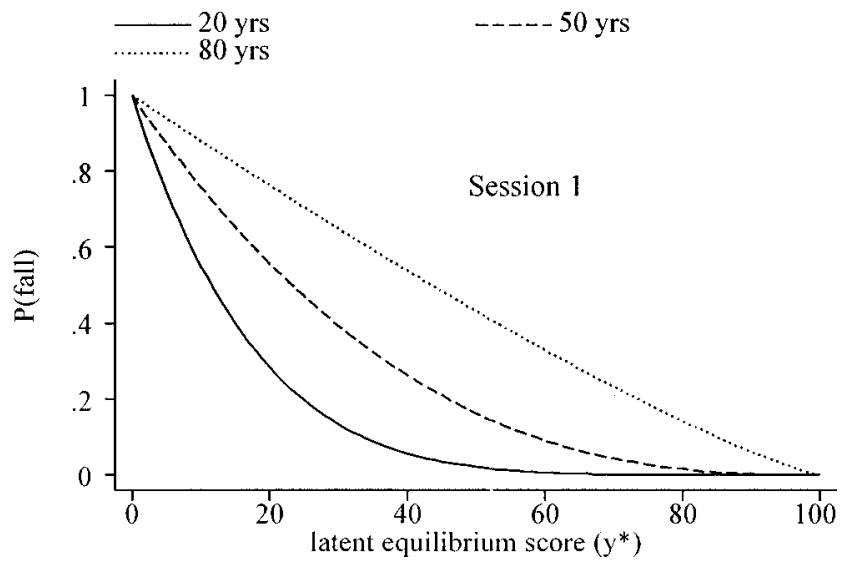

(a)

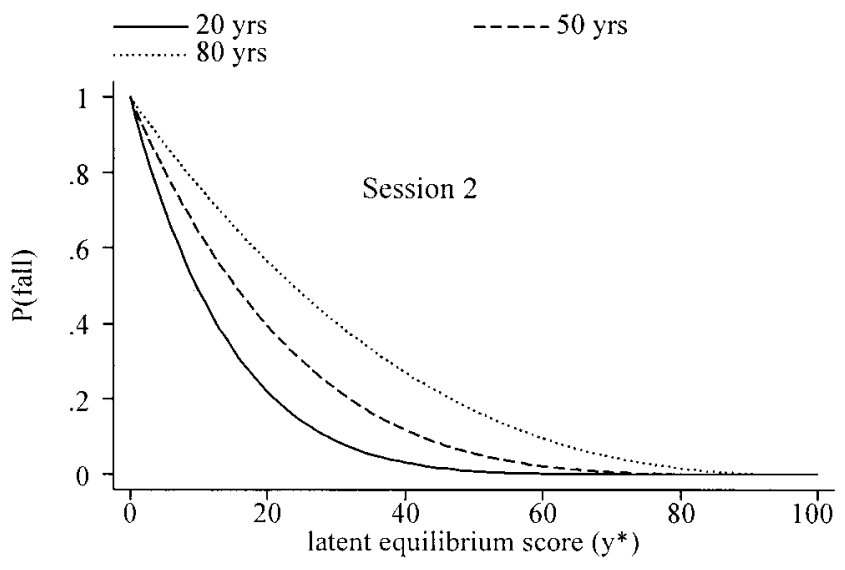

(b)

Fig. 7. Estimated conditional probability of a fall given latent ES. Ages 20, 50, and 80 years. (a) Session 1. (b) Session 2.

the first session $\left(\gamma_{2}<0 ; P<0.001\right)$. Fig. 6 shows the estimated value of $r$ as a function of age and session (lower curve $=$ Session 1, upper curve $=$ Session 2). This effect of age and session on $r$ in turn affects the probability of a fall as shown in Fig. 7 for average subjects aged 20, 50, and 80 yrs. However, age had no direct effect on the distribution of the latent ES itself, being removed from the equations for $\log p$ and $\log q$ after showing no significant effect. The implication is that older subjects tend to sway as much as younger ones, but are more likely to fall for a given amount of sway. To see this graphically, 


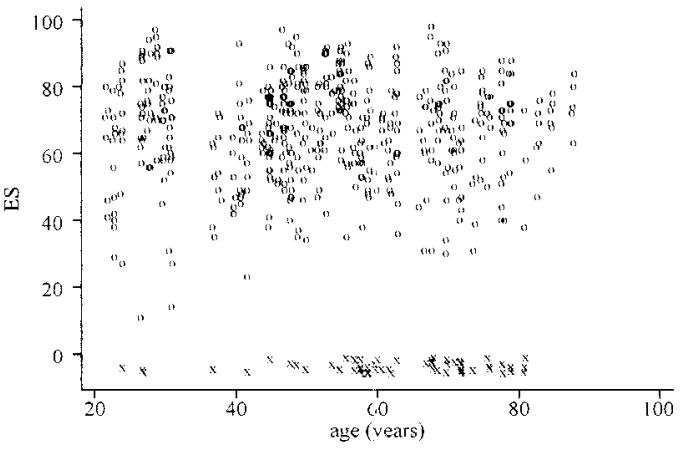

(a)

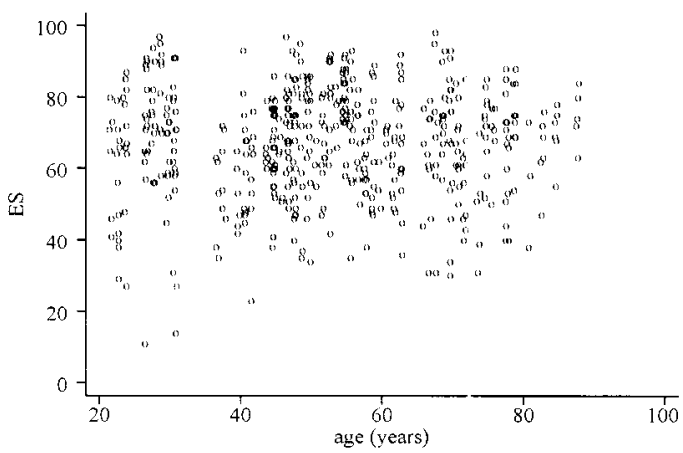

(b)

Fig. 8. Distribution of observed ES by age. (a) Falls (shown as " $x$ ") are coded as zero with small random negative perturbations for visibility. (b) Falls are removed.

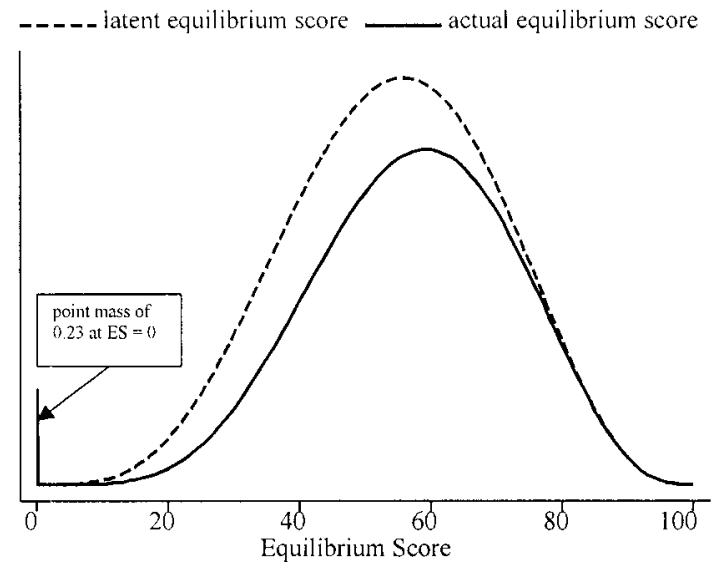

Fig. 9. Estimated probability distributions of latent and actual ES.

consider the scatter plot of ES vs. age with falls coded as zero [Fig. 8(a)]. Note that although most of the falls were recorded by older subjects, the lowest nonfall ESs were observed on younger subjects. This counter-intuitive phenomenon is explained by all subjects having the same distribution of latent ESs, but that for low values of latent ES, older subjects were much more likely to fall, hence, there are few, if any, observed low ESs for older subjects when falls are removed [Fig. 8(b)].

\section{DISCUSSION}

We have presented a methodology for analyzing the effect of experimental variables on CDP test ESs, taking into account the skewed distribution of these scores and the possibility of falls. For a given covariate pattern, our method estimates the

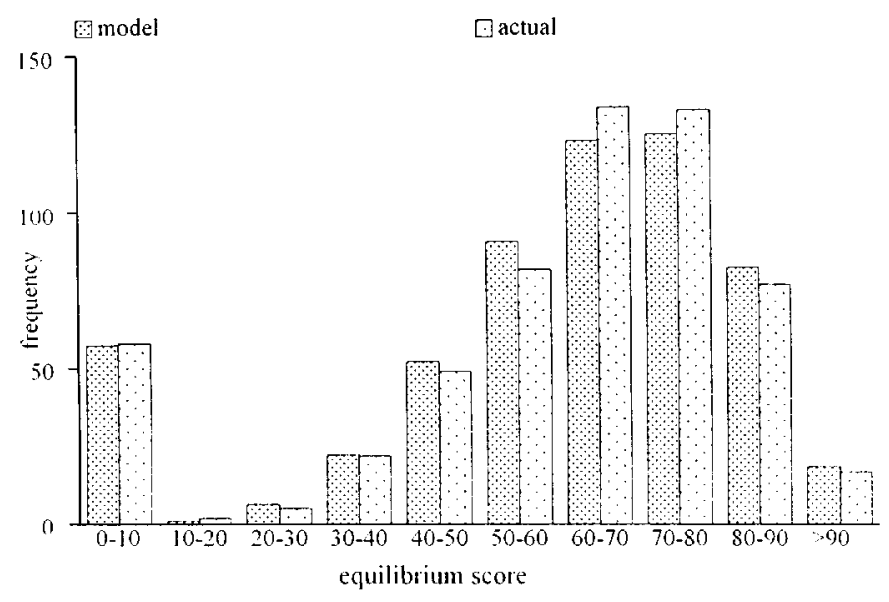

Fig. 10. Goodness of fit for BLSA data. Model-predicted versus actual number of ES cases falling into ten equally spaced bins. There were a total of 579 cases overall.

distribution of the latent ES, which is a continuous extension of the standard score to cases of poor balance control where falls are likely. Because the latent score always exists (although it is not always observed) it makes sense to calculate the mean or any other characteristic of its probability distribution. From this distribution, integrated with the fall model though $G(y)$, one may calculate (for the same covariate pattern) properties of the corresponding mixed discrete-continuous distribution of actual ESs, where falls are counted as zeros. As an illustration of the difference between latent and actual ESs, Fig. 9 shows plots of the distributions of estimated latent and actual ESs for a typical 60-year-old subject on Session 1, Trial 1 of the BLSA study. The difference in the areas under the continuous parts is exactly the probability of a fall (0.23), which is the amount of point mass at zero for the actual ES.

\section{A. Goodness of Fit}

To see if our model was indeed realistic for the BLSA data, we compared the number of occurrences of trials with ESs lower than preset thresholds with corresponding model-predicted frequencies. Because of the varied covariate patterns (variety of sessions, trials, and subject ages) in the BLSA data, the model predictions could not be obtained from one particular scaled beta distribution. However we were able to estimate the distribution of $Y$ for the actual mix of covariate patterns present. For a fixed covariate pattern with falls coded as zeros, the probability of an actual ES equal to or lower than a given value $y_{0}$ in $(0,100)$ is

$$
P\left(Y \leq y_{0}\right)=\pi+P\left(Y \leq y_{0} \mid \text { no fall }\right)(1-\pi) .
$$

Since the conditional distribution of $Y$ given no fall has density $f_{0}(y)=(1-G(y)) f(y) /(1-\pi)$, it follows that

$$
\begin{aligned}
P\left(Y \leq y_{0}\right)=\pi+\frac{\Gamma(p+q)}{\Gamma(p) \Gamma(q)}\left[\int_{0}^{y_{0} / 100} z^{p-1}(1-z)^{q-1} d z\right. \\
\left.-\int_{0}^{y_{0} / 100} z^{p-1}(1-z)^{r+q-1} d z\right] \\
=\pi+I\left(p, q, \frac{y_{0}}{100}\right)-\frac{\Gamma(p+q) \Gamma(r+q)}{\Gamma(q) \Gamma(r+p+q)} I\left(p, r+q, \frac{y_{0}}{100}\right)
\end{aligned}
$$


where $I(p, q, y)$ is the incomplete Beta function and the parameters $p, q$ and $r$, depend on the covariate pattern through a relationship such as the one given by (7). Summing the estimated value of (9) over all the observations in our data set gives an estimate of the number of observations such that $Y \leq y_{0}$. This model-predicted unconditional cumulative distribution can be differenced to obtain the expected number of ES scores falling into an arbitrary interval. As an example, Fig. 10 shows the expected and actual number of ES scores in ten equally spaced bins between 0 and 100 .

\section{B. Alternative Models}

In the course of this study, we considered several other models for explaining falls as cases of being unable to completely observe some quantitative aspect of balance control-in this study, $Y^{*}$, the "latent ES." One candidate for an alternative model is that of "censoring." In its simplest form, a censoring model would state that when a fall occurs, we would only know that $Y^{*}$ is less than a known cutoff value. In our scenario, we do not have explicit cutoff values, but we might assume, for example, that falls always occur if $Y^{*}<9$ (or some other value less than the minimum of observed nonzero ESs). The main problem with the censoring approach is that $Y^{*}$ is only an imperfect measure of balance control. For this reason, it would be over-presumptive to state that falls would definitely occur or not occur depending on the value of $Y^{*}$. Additional aspects of balance control other than peak-peak sway would also influence whether or not a fall occurs. Therefore, it would be more realistic to say that the value of $Y^{*}$ only determines the degree to which the event of a fall is likely or unlikely. An examination of the data for which low nonzero values of $Y$ are rare, but that falls are not, supports the notion of a fall mechanism not entirely manifested in values of $Y^{*}$.

Another statistical model that attempts to account for nonresponse is the Heckman selection model [20], [21], commonly found in applications of econometrics. Heckman's model assumes a normal distribution for the latent response variable whose mean and probability of nonresponse are determined by separate linear combinations of explanatory variables. Although ESs are restricted in range, Heckman's model might be effective on a transformed score such as $Y^{\prime}=\log (Y / 100-Y)$, which is closer to being normally distributed than $Y$ (although other problems remain-see Section I-B). For a given covariate pattern, the Heckman model also allows for the possibility of correlation between the latent response and a nonresponse indicator variable. In our situation, we incorporate this dependence directly into the model by specifying the conditional distribution of $\phi$ given $Y^{*}$. Unlike the Heckman model, which assumes constant correlation, our framework allows the correlation to depend on the experimental variables through the linear equation for $\log r$. Also, by conditioning on $Y^{*}$, we incorporate a dependence of the fall probability on the actual test performance, as well as the independent variables. In the Heckman model, the dependence is only in terms of the independent variables, which may reflect expected, but not actual performance.

The use of the form $G\left(Y^{*}\right)=\left(1-Y^{*}\right)^{r}$ for $P\left(\phi=1 \mid Y^{*}\right)$ is a mathematical convenience allowing the evaluation of relatively simple expressions for $\pi=P($ fall $)$ and the likelihood function. However the shape of $G\left(Y^{*}\right)$ is appropriate, reflecting the increased risk of fall as sway increases (decreasing ES). For the amount of falls present in the BLSA data, we feel that using $\left(1-Y^{*}\right)^{r}$ approximates the conditional risk of falling as well as any reasonable alternative would. Under our model, it can be shown that $\operatorname{Cov}\left(\phi, Y^{*}\right)=100 p \pi(1 / p+q+r-1 / p+q)$, $\operatorname{Var}(\phi)=\pi(1-\pi)$, and $\operatorname{Var}\left(Y^{*}\right)=10^{4} p q /(p+q)^{2}(p+q+I)$. From these, we calculated the correlation between $\phi$ and $Y^{*}$ averaged over all observations to be about -0.27 . As a check, we fit Heckman's model to our data and estimated the average correlation (assumed to be a constant) to be about -0.33 . The discrepancy between these two quantities is well within the expected margin of error.

Although our model allows for both $\log p$ and $\log q$, to be expressed by potentially all independent variables, we found in practice that including a given predictor in both equations only trivially improved the overall fit. We, therefore, restricted each predictor variable to appear in no more than one of the equations. However we did allow any of the predictors to appear in the equation for $\log r$. Our final choice of predictors, shown in (7), resulted from finding the allocation to the $\log p$ and $\log q$ equations which gave the best overall fit in terms of $\log$ likelihood. For example, the value of the log likelihood corresponding to the model given by (7) was 129.33 . For a similar set of equations with the predictors for $\log p$ and $\log q$ switched, the value of the log likelihood was 127.29.

\section{Conclusion}

Assessment of balance control function by CDP has become standard practice for neuro-otologists, neurologists, gerontologists, occupational and physical therapists, space physiologists, and other specialists (see [1] for an overview). Studies by these specialists frequently seek to quantify the deleterious effects on balance control function caused by pathologic, demographic, and/or environmental factors or the salutary effects achieved by therapeutic interventions. Accurate evaluation of these withinand between-subject study design protocols depends on statistical analysis of the CDP dependent measure (ES) for all subjects and conditions. Such analyses are hampered by the discontinuous nature of the ES when the test subject loses balance. The technique presented here provides for the first time an objective method for incorporating fall data into statistical analysis of CDP data sets. Previous approaches had significant shortcomings: Excluding falls from data sets leads to an underestimation of the true stability level of a population, while coding falls as zeros leads to violations of distributional assumptions underlying the use of traditional statistical tests.

Equilibrium scores from the CDP platform follow a paradigm in which: 1) the observed data (EQ scores) provides surrogate information on a unobservable characteristic of interest (the latent EQ, or capacity for balance control); 2) the process through which the surrogate information is obtained is subject to random failure (falls); and 3) the probability of such failures depends functionally on the unobserved characteristic (the latent EQ). Using similar methodology (but perhaps with different distributional models) data from other situations falling under this paradigm could be interpreted without resorting to ignoring failures or to conventional analysis methods after arbitrary coding of failures. In particular, we feel that our methodology could be applied to the following situations. 
1) Urinalysis Test Strips. If a person's blood glucose is above a certain threshold, excess sugar will be excreted in urine and, hence, be easily detected with a glucose reagent strip [22]. The color of the strip can be approximately correlated with blood glucose level. Conversely, for blood sugar levels less than the threshold, the reagent strip would show nothing (a "nonresponse"). Because the threshold value varies randomly over individuals, there is a functional dependence between blood sugar level and the probability of nonresponse, which can be exploited to reconstruct the distribution of actual blood sugar over a subpopulation of subjects, e.g., those following a certain treatment regimen. The reagent strip is a surrogate for the more time-consuming and inconvenient direct analysis of blood samples.

2) Detection of Patent Foramen Ovale (PFOs). A PFO is a congenital hole in the septum, i.e., the wall separating the right and left atria in the heart. This defect is found in approximately $27.5 \%$ of adult humans [23]. A common method for detecting and assessing the size of PFOs is contrast-enhanced transthoracic echocardiography (c-TTE). The method works by using an external chest probe to detect bubbles in the left atrium after injection of an echo contrast agent in a peripheral vein [24], [25]. In general, the presence of bubbles in the left atrium signals a PFO, with larger PFOs being associated with more bubbles. However, because remote views of the heart from the chest may be perturbed, there is the possibility of no bubbles being detected (a "failure") even if an actual PFO is present. Nevertheless, it is reasonable to assume the probability of observing no bubbles is functionally dependent on the actual PFO size, thus our nonresponse model would apply.

Of course, the degree to which our approach would be effective for other applications remains to be verified; however in the case of equilibrium scores, we have shown that our methodology is an effective analysis tool that should prove useful in both research and clinical settings.

\section{REFERENCES}

[1] L. M. Nashner, "Computerized dynamic posturography," in Handbook of Balance Function Testing, GP Jacobsen, CW. Newman, and JM. Kartush, Eds. Chicago, IL: Mosby-Year Book, 1993, p. 309 and p. 323.

[2] M. Asai, Y. Watanabe, N. Ohashi, and K. Mizukoshi, "Evaluation of vestibular function by dynamic posturography and other equilibrium examinations," Acta Otolaryngol (Stockh), vol. Suppl 504, pp. 120-124, 1993.

[3] W. H. Paloski, F. O. Black, M. F. Reschke, D. S. Calkins, and C. S. Shupert, "Vestibular ataxia following shuttle flights: Effects of microgravity on otolith mediated sensorimotor control of posture," Amer. J. Otol., vol. 14, no. Number 1, pp. 12-15, 1993.

[4] C. R. Fox and G. D. Paige, "Effect of head orientation on human postural stability following unilateral vestibular ablation," J. Vestib. Res., vol. 1, pp. 155-156, 1990-1991.

[5] S. L. Zeger, K. Y. Liang, and P. S. Albert, "Models for longitudinal data: A generalized estimating equation approach," Biometrics, vol. 44, pp. 1049-1060, 1988.

[6] P. J. Huber, "The behavior of maximum-likelihood estimates under nonstandard conditions," in Proc. 5th Berkeley Symp. Mathematical Statistics and Probability, Berkeley, CA, 1967, pp. 221-233.

[7] R. M. Royall, "Model robust confidence intervals using maximum likelihood estimators," Int. Statist. Rev., vol. 54, pp. 221-226, 1986.
[8] Neurocom System Operator's Manual Version 4.04, Neurocom International, Inc, Clackamas, OR, 1992, pp. F-6-F-7.

[9] R. J. Peterka and F. O. Black, "Age related changes in human posture control: Sensory organization tests," J. Vestib. Res., vol. 1, p. 81, 1990.

[10] A. Rupert, (personal communication), Naval Aerosp. Med. Res. Lab., Pensacola, FL.

[11] W. H. Paloski, M. F. Reschke, F. O. Black, and R. S. Dow, "Recovery of postural equilibrium control following space flight," Extended Duration Orbiter Medical Project, NASA, Lyndon B. Johnson Space Center, Houston, TX, Tech. Rep. NASA SP 1999-534, C. F. Sawin, G. R. Taylor, and W. L. Smith, Eds., 1999.

[12] R. J. Peterka and F. O. Black, "Age related changes in human posture control: Sensory organization tests," J. Vestib. Res., vol. 1, p. 80, 1990.

[13] T. Ledin, A. C. Kronhed, C. Moller, M. Moller, L. M. Odkvist, and B. Olsson, "Effects of balance training in elderly evaluated by clinical tests and dynamic posturography," J. Vestib. Res., vol. 1, p. 132, 1990-91.

[14] N. T. Shepard, A. Schultz, M. J. Gu, N. B. Alexander, and T. Boismier, "Postural control in young and elderly adults when stance is challenged: Clinical versus laboratory measurements," Ann. Otol., Rhinol., Laryngol., vol. 102, no. 7, pp. 509-515, 1993.

[15] R. J. Serfling, Approximation Theorems of Mathematical Statistics. New York: Wiley, 1980, pp. 243-261.

[16] I. Domowitz and H. White, "Misspecified models with dependent observations," J. Econometrics, vol. 20, pp. 35-58, 1982.

[17] Stata Statistical Software, Version 7.0, Stata Corp, College Station, TX, 2001.

[18] W. Gould and W. Sribney, Maximum Likelihood Estimation With Stata. College Station, TX: Stata, 1999, pp. 9-10.

[19] N. W. Shock, R. C. Gruelich, and R. A. Andres et al., Normal Human Aging. The Baltimore Longitudinal Study of Aging. Washington, DC: U.S. Government Printing Office, 1984.

[20] J. Heckman, "Sample selection bias as a specification error," Econometrica, vol. 47, pp. 153-161, 1976

[21] W. H. Greene, Econometric Analysis, 4th ed. Englewood Cliffs, NJ: Prentice-Hall, 2000.

[22] J. B. Henry et al., Clinical Diagnosis and Management by Laboratory Methods, 18th ed. Philadelphia, PA: Saunders, 1991, p. 396, 397, and 415.

[23] P. T. Hagen, D. G. Scholz, and W. D. Edwards, "Incidence and size of patent foramen ovale during the first 10 decades of life: An autopsy study of 965 normal hearts," Mayo Clin. Proc., vol. 59, pp. 17-20, 1984.

[24] P. Siostrzonek, M. Zangeneh, and H. Gossinger et al., "Comparison of transesophageal and transthoracic contrast echocardiography for detection of a patent foramen ovale," Amer. J. Cardiol., vol. 68, pp. 1247-9, 1991.

[25] R. N. Belkin, B. D. Pollack, and M. L. Ruggiero et al., "Comparison of transesophageal and transthoracic echocardiography with contrast and color flow doppler in the detection of patent foramen ovale," Amer. Heart J., vol. 128 , pp. 520-5, 1994.

Alan H. Feiveson is a Statistician working with the NASA Johnson Space center as a consultant in the application of statistics to a variety of NASA projects from 1964 to the present. From 1984 to 1995, he consulted on engineering studies of the aerodynamic effects on the Space Shuttle during ascent into orbit and also on various Shuttle reliability studies. From 1995 to the present, he has provided statistical consultation for a number of research projects aimed at finding countermeasures to the adverse effects of spaceflight on the human physiology. During this time, he has co-authored a number of papers dealing with applications of statistics to NASA-related research in neurology, orthopedics, environmental physiology, and microbiology.

E. Jeffrey Metter is a Neurologist currently with the National Institute of Aging, Intramural Research Program in the Laboratory of Clinical Investigation, Gerontology Research Center, Baltimore, MD. He is a physician working with the Baltimore Longitudinal Study of Aging, and has been with the study since 1987.

His research focuses on human aging and diseases associated with getting older. He works in several areas including age-associated changes in nerve, muscle, and physical activity. 
William H. Paloski (S'79-M'82) was trained as a biomedical engineer

He has been with the Johnson Space Center for more than 15 years. He is currently Chief of the Human Adaptation and Countermeasures Office, where he is responsible for managing the Intramural Life Sciences Research Laboratories and the Clinical/Operational Research Program, which includes the Countermeasure Evaluation and Validation Project. He is also a Senior Scientist in the Neurosciences Laboratory. His research includes operational studies of postural stability, control, and performance before and after space flight and basic studies of neuro-vestibular adaptation to altered gravito-inertial environments (specifically aimed toward development of prescriptions for use of Artificial Gravity as a countermeasure). He has published and/or presented more than 100 research articles.

Dr. Paloski is a member of the Barany Society and Sigma Xi, the Scientific Research Society. 